\title{
ON GRADIENT MAPPINGS IN BANACH SPACES
}

\author{
TSUYOSHI ANDô
}

1. Introduction. Let $E$ be a real Banach space, $V$ a convex open subset of $E$. A real valued functional $I(x)$ defined on $V$ is said to have the Fréchet derivative $D(x, h)$ if, for arbitrary fixed $x \in V, D(x, h)$ is a bounded linear functional on $E$ in the variable $h$ and

$$
I(x+h)-I(x)=D(x, h)+\omega(x, h)
$$

where

$$
\omega(x, h)=o(\|h\|) .
$$

Thus $x \rightarrow D(x, \cdot)$ is a mapping of $V$ into the space $E^{*}$ conjugate to $E$, and is called a gradient mapping. It is said to be compact, if the image of each bounded set of $V$ has a compact closure in $E^{*}$.

Recently E. H. Rothe [1] gave a (necessary and) sufficient condition for the compactness of gradient mappings under the condition that $E$ has property $(P)$, and showed that every reflexive Banach space with a basis has it. Here $E$ is said to have property $(P)$, if there exists a sequence $\left\{\psi_{i}\right\}$ of linearly independent elements of $E^{*}$ and a positive number $M$ with the following properties: the closed linear span of $\left\{\psi_{i}\right\}$ coincides with $E^{*}$ and for each positive integer $n$ there exists a linear projection of norm at most $M$ on the intersection $\bigcap_{i=1}^{n} N_{i}$ where $N_{i}=\left\{x \in E \mid \psi_{i}(x)=0\right\}$. In this note a theorem with the conclusion that the gradient mapping is compact is proved under conditions differing somewhat from those of Rothe, and without using anything like the property $(P)$.

TheOREM. Let $I(x)$ have the following two properties: (a) for each $\alpha>0$ there exists a positive number $\beta$ such that

$$
|I(x)-I(y)| \leqq \beta\|x-y\|, \quad x, y \in V,\|x\|,\|y\| \leqq \alpha ;
$$

(b) to each $\epsilon>0$ corresponds a finite number of elements $\phi_{1}, \phi_{2}, \cdots, \phi_{n}$ of $E^{*}$ such that

$$
|I(x+h)-I(x)| \leqq \epsilon\|h\|, \quad x, x+h \in V,
$$

for all $h$ for which $\|h\| \leqq \epsilon$ and $\phi_{i}(h)=0(i=1,2, \cdots, n)$. Then the gradient mapping $D(x, \cdot)$ is compact.

2. Proof of theorem. Our proof is based on the following decomposition lemma instead of on the use of linear projections.

Received by the editors April 18, 1960. 
LemMa. Let $N$ be a closed linear subspace of $E$ with a finite co-dimension. Then there exists a compact set $A$ with the properties that $\|x\|$ $\leqq 1(x \in A)$, and each $h \in S$ (the open unit ball) admits a decomposition (not unique) $h=a+b$ with $a \in A, b \in N$.

Proof. We consider as usual the quotient space $E / N$ with the norm defined by $\|P(x)\|=\inf _{y \in N}\|x-y\|$ where $x \rightarrow P(x)$ is the canonical mapping of $E$ onto $E / N$. Since $E / N$ is finite dimensional, the image $P(S)$ of $S$ has a compact closure in it. We shall construct by induction a sequence $\left\{a_{i j}\right\}, i=0,1,2, \cdots, j=1,2, \cdots, k_{i}$, of elements of $S$ such that

(2) $\min _{k}\left\|a_{i k}-a_{i+1, j}\right\|<1 / 2^{i}, \quad i=0,1,2, \cdots, j=1,2, \cdots, k_{i+1}$,

and for each $x \in S$ and each $i$ there exists $y \in E$ (depending on $x$ and i) such that

$$
P(x)=P(y) \text { and } \min _{j}\left\|a_{i j}-y\right\|<1 / 2^{i} .
$$

Put $k_{0}=1$ and $a_{01}=O$ (the origin). Suppose that

$$
\left\{a_{i j}\right\}, \quad i=0,1,2, \cdots, n, \quad j=1,2, \cdots, k_{i},
$$

have been constructed. Denote, for convenience, by $S_{n}(x)$ the open ball with center $x$ and radius $1 / 2^{n}$. Since $P\left(S_{n}\left(a_{n j}\right) \cap S\right)$ is totally bounded in $E / N$ and $P(S) \subset U_{j} P\left(S_{n}\left(a_{n j}\right)\right)$ because of (3), there exists a finite number of elements of $S$, denoted by $a_{n+1, j}, j=1,2, \cdots, k_{n+1}$, such that $a_{n+1, j} \in \mathrm{U}_{k} S_{n}\left(a_{n k}\right), j=1,2, \cdots, k_{n+1}$, and

$$
\min _{j}\left\|P(x)-P\left(a_{n+1, j}\right)\right\|<1 / 2^{n+1} \quad \text { for all } x \in S \text {. }
$$

Then on account of the definition of norm in $E / N$, for each $x \in S$ there can be chosen $y \in E$ such that $P(x)=P(y)$ and $\min _{j}\left\|a_{n+1, j}-y\right\|$ $<1 / 2^{n+1}$. Thus the induction is completed. Notice that the set $\left\{P\left(a_{i j}\right)\right\}$ is dense in $P(S)$ by consiruction. We claim that the closure $A$ of the set $\left\{a_{i j}\right\}$ is compact. In fact, from (2) it follows that for each $n, m$ with $m \geqq n$

$$
\min _{k}\left\|a_{m j}-a_{n k}\right\|<\sum_{i=n}^{m} 1 / 2^{i}<1 / 2^{n-1} .
$$

Since $\left\{a_{i j} \mid i \leqq n\right\}$ is a finite set, this means that the set $\left\{a_{i j}\right\}$ is totally bounded; consequently its closure $A$ is compact. Since $P(A)$ is compact in $E / N$ and contains a dense subset of $P(S)$, it follows that $P(A) \supset P(S)$, that is, for each $h \in S$ there exists $a \in A$ with $P(a)$ $=P(h)$, i.e., $h-a \in N$. 
Now we turn to the proof of the theorem. If $D(x, \cdot)$ is not compact, there exist a positive number $\epsilon$ and a sequence $\left\{x_{i}\right\}$ of elements of $V$ such that $\left\|x_{i}\right\| \leqq 1 / \epsilon, i=1,2, \cdots$, and $\left\|D\left(x_{i}, \cdot\right)-D\left(x_{j}, \cdot\right)\right\|>\epsilon$ for $i \neq j$. Let $\phi_{1}, \phi_{2}, \cdots, \phi_{n}$ be chosen as in (b) with $\epsilon / 8$ instead of $\epsilon$. Since $N=\left\{x \in E \mid \phi_{i}(x)=0, i=1,2, \cdots, n\right\}$ has a finite co-dimension, by the lemma there exists a compact set $A$ of $S^{-}$(the closure of $S$ ) such that each $h \in S$ admits a decomposition $h=a+b$ with $a \in A$, $b \in N$. It follows that $\|b\| \leqq\|a\|+\|h\| \leqq 2$. Since property (a) combined with definition (1) implies $\sup _{i}\left\|D\left(x_{i}, \cdot\right)\right\|<\infty$, the family $\left\{D\left(x_{i}, h\right)\right\}$, considered as continuous functions on the compact set $A$ in the variable $h$, is equi-continuous; consequently Ascoli-Arzela's theorem tells us that there exists a subsequence $\left\{x_{i}^{\prime}\right\}$ for which

$$
\sup _{a \in A}\left|D\left(x_{i}^{\prime}, a\right)-D\left(x_{j}^{\prime}, a\right)\right|<\epsilon / 2, i, j=1,2, \cdots .
$$

On the other hand, on account of the definition of norm of linear functionals,

$$
\begin{aligned}
& \left\|D\left(x_{i}^{\prime}, \cdot\right)-D\left(x_{j}^{\prime}, \cdot\right)\right\|=\sup _{h \in S}\left|D\left(x_{i}^{\prime}, h\right)-D\left(x_{j}^{\prime}, h\right)\right| \\
& \quad \leqq \sup _{a \in A}\left|D\left(x_{i}^{\prime}, a\right)-D\left(x_{j}^{\prime}, a\right)\right|+2 \cdot \sup _{k ; b \in N,\|b\| \leqq 2}\left|D\left(x_{k}^{\prime}, b\right)\right| .
\end{aligned}
$$

Since $b \in N$ and $\|b\| \leqq 2$, property (b) (with $\epsilon / 8$ ) combined with (1) implies $\left|D\left(x_{k}^{\prime}, b\right)\right| \leqq \epsilon / 4$, consequently

$$
\left\|D\left(x_{i}^{\prime}, \cdot\right)-D\left(x_{j}^{\prime}, \cdot\right)\right\| \leqq \epsilon / 2+\epsilon / 2=\epsilon .
$$

This contradiction establishes the theorem.

\section{REFERENCE}

1. E. H. Rothe, $A$ note on gradient mappings, Proc. Amer. Math Soc. vol. 10 (1959) pp. 931-935.

HOKKAIDO UNIVERSITY,

SAPPORO, JAPAN 